\title{
Ultra-low-cost near-infrared photodetectors on silicon
}

\author{
M. Amin Nazirzadeh ${ }^{\mathrm{a}, \mathrm{b}}$, Fatih B. Atar, ${ }^{\mathrm{a}, \mathrm{b}}$, B. Berkan Turgut ${ }^{\mathrm{a}, \mathrm{b}}$, Ali K. Okyay*a,b,c \\ ${ }^{\mathrm{a} D e p a r t m e n t ~ o f ~ E l e c t r i c a l ~ a n d ~ E l e c t r o n i c s ~ E n g i n e e r i n g, ~ B i l k e n t ~ U n i v e r s i t y, ~ B i l k e n t, ~ A n k a r a, ~ T u r k e y ; ~}$ \\ ${ }^{b}$ National Nanotechnology Research Center (UNAM), Bilkent University, Bilkent, Ankara, Turkey; \\ ${ }^{\mathrm{c}}$ Institute of Materials Science and Nanotechnology, Bilkent University, Bilkent, Ankara, Turkey
}

\begin{abstract}
We demonstrate Silicon-only near-infrared (NIR) photodetectors (sensitive up to $2000 \mathrm{~nm}$ ) that meet large-scale ultralow-cost fabrication requirements. For the detection of infrared photons, we use metal nanoislands that form Schottky contact with Silicon. NIR photons excite plasmon resonances at metal nanoislands and plasmons decay into highly energetic charge carriers (hot electrons). These hot electrons get injected into Silicon (internal photoemission), resulting in photocurrent. Several groups have studied plasmonic nanoantennas using high resolution lithography techniques. In this work, we make use of randomly formed nanoislands for broad-band photoresponse at NIR wavelengths. We observe photoresponse up to $2000 \mathrm{~nm}$ wavelength with low dark current density about $50 \mathrm{pA} / \mu \mathrm{m}^{2}$. The devices exhibit photoresponsivity values as high as $2 \mathrm{~mA} / \mathrm{W}$ and $600 \mu \mathrm{A} / \mathrm{W}$ at $1.3 \mu \mathrm{m}$ and $1.55 \mu \mathrm{m}$ wavelengths, respectively. Thin metal layer was deposited on low-doped n-type Silicon wafer. Rapid thermal annealing results in surface reconstruction of the metal layer into nanoislands. Annealing conditions control the average size of the nanoislands and photoresponse of the devices. An Al-doped Zinc Oxide (AZO) layer was deposited on the nanoislands using thermal atomic layer deposition (ALD) technique to acts as a transparent conductive oxide (TCO) and patterned using photolithography. AZO film creates electrical connection between the nanoislands and also makes a heterojunction to Silicon. Simple and scalable fabrication on $\mathrm{Si}$ substrates without the need for any sub-micron lithography or high temperature epitaxy process make these devices good candidates for ultra-low-cost broad-band NIR imaging and spectroscopy applications.
\end{abstract}

Keywords: Silicon, Plasmonics, hot electrons, infrared detectors, Schottky diodes.

\section{INTRODUCTION}

Sub-bandgap photodetection via the internal photoemission mechanism is an attractive candidate for near-infrared (NIR) photodetection on Si. A metal in contact with Si creates a Schottky junction and a potential energy barrier forms between the metal and $\mathrm{Si}$. This junction operates as a diode (Schottky diode) and can be used as a sub-bandgap photodetector. The sub-bandgap photons incident on the metal layer excites the electrons of the metal to higher energy levels, enabling them to traverse the Schottky barrier and be collected as photocurrent ${ }^{1}$. The interest in this approach has received a significant boost with the recent studies on the use of metallic nanoantennas to capture the incident light by exciting surface plasmons $^{2-4}$.

Metals are normally highly reflective at NIR wavelengths and can poorly absorb the incident light. This had been the main drawback of using Si Schottky barrier diodes for NIR photodetection. However, when made into nanostructures, some metals, such as $\mathrm{Au}$, can resonantly interact with the incident light and confine the electromagnetic field in very small volumes in the form of surface plasmons. The surface plasmons decay due to the optical losses of the metal and as they decay their energy is transferred to the electrons of the metal, exciting the electrons to higher energy levels, which are now called "hot electrons". These hot electrons can be emitted over the Schottky barrier and generate photocurrent ${ }^{2}$.

*aokyay@ee.bilkent.edu.tr

Silicon Photonics X, edited by Graham T. Reed, Michael R. Watts, Proc. of SPIE Vol. 9367 ,

93671I · C 2015 SPIE · CCC code: 0277-786X/15/\$18 · doi: 10.1117/12.2078913 


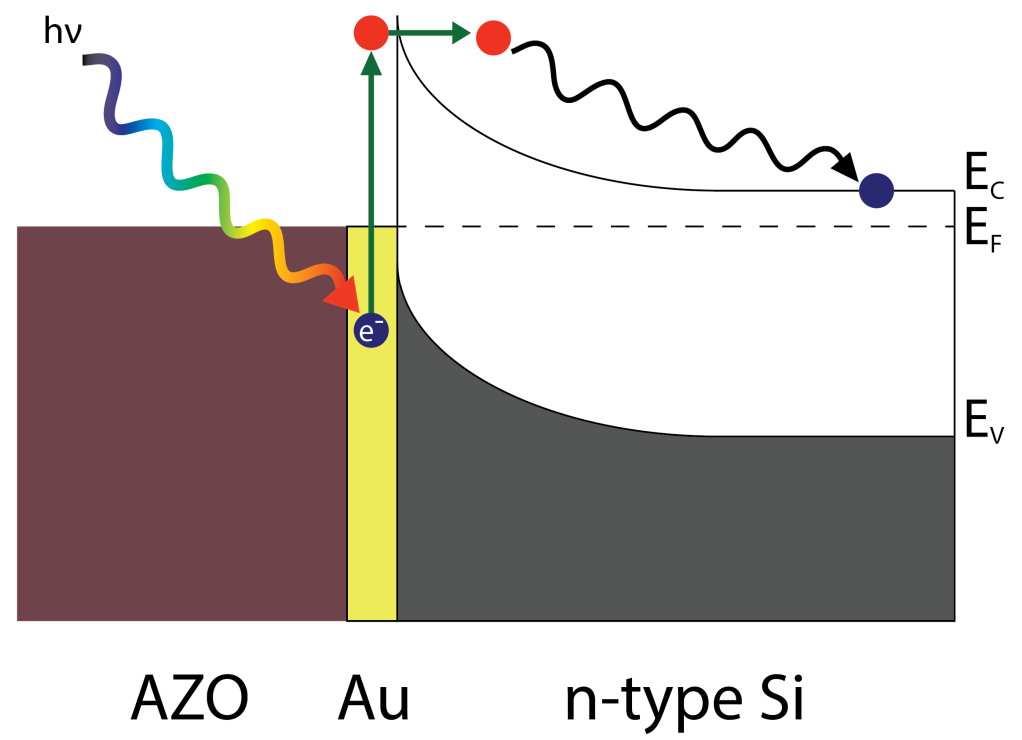

Figure 1. Energy band diagram of a Schottky junction. Photodetection of a sub-bandgap photon via the internal photoemission is shown on the energy band diagram ${ }^{1}$.

Several studies have investigated the plasmon assisted hot electron generation process and its utilization for NIR photodetectors by using high resolution lithography techniques to fabricate metallic nanoantennas ${ }^{2,3}$. Very strong, tunable, narrow-band plasmonic resonances have been demonstrated and photoresponsivity values as high as $0.6 \mathrm{~mA} / \mathrm{W}$ have been reported ${ }^{3}$. In this study, we first investigate the fabrication process of metallic nanoantennas and propose a method to form randomly sized and randomly distributed nanoantennas on Si surface. We then demonstrate the use of these random nanoantennas in a Schottky contact photodetector for broad-band plasmon enhanced NIR photodetection.

\section{FABRICATION}

We used 4-inch n-Si (100) wafers with resistivity of 2-5 $\Omega$-cm throughout this study and diced the wafers into $15 \mathrm{~mm} \times$ $15 \mathrm{~mm}$ pieces prior to fabrication. Cleaning of the wafers was done in two consecutive steps. In the first step, the wafers are kept in piranha solution $\left((4: 1) \mathrm{H}_{2} \mathrm{SO}_{4}: \mathrm{H}_{2} \mathrm{O}_{2}\right)$ at $80^{\circ} \mathrm{C}$ for 5 minutes to remove possible organic and metallic contaminants. Piranha solution also oxidizes the wafer surface and forms a thin $\mathrm{SiO}_{2}$ layer, making the surface hydrophilic. The wafers are rinsed with deionized water after the piranha cleaning. In the second step of the cleaning process, the wafers are dipped in buffered hydrofluoric acid (BHF) solution for about 10 seconds until the surface oxide was completely etched and the sample surface became hydrophobic again. The wafers are then rinsed with DI water and dried with $\mathrm{N}_{2}$ gun.

We have followed several approaches to form plasmonic nanoantennas on Si surface. Electron beam lithography and nanoimprint lithography were used to fabricate nanoantennas with desired dimensions. A third method, involving annealing of a metal layer to form randomly shaped nanoislands, was also investigated.

Nanoimprint lithography is a low-cost, fast, and high resolution lithography method. The desired pattern is first fabricated on a master stamp using a high resolution lithography technique. The pattern on the master stamp is then transferred to a mold which is Polydimethylsiloxane (PDMS) for our case. PDMS consists of a base Silicone elastomer and its corresponding curing agent. The base and the curing agent are mixed carefully in 10:1 volume ratio for about 10 minutes to achieve uniform distribution in the mixture. The mixture is then kept in a desiccator for an hour to remove the air bubbles that may form during the mixing process. The PDMS mixture is poured on the master stamp and cured at $150^{\circ} \mathrm{C}$. The PDMS mold takes the shape of the master stamp and must be peeled off carefully after the curing process. The pattern on the PDMS mold is transferred to the sample by coating a photoresist layer on the sample and pressing the PDMS mold on the photoresist. The pressure is applied conformally and at a temperature higher than the glass transition temperature of the photoresist. The photoresist takes the shape of the stamp and hardens with this process. A final dry etching step is applied to remove the residual photoresist. The flow diagram of the nanoimprint lithography process is 
shown in Fig. 2. The scanning electron microscope (SEM) image of the samples after nanoimprint lithography process is shown in Fig. 3(a).

a)

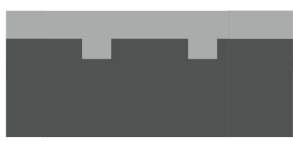

Photoresist PDMS mold Substrate

c)

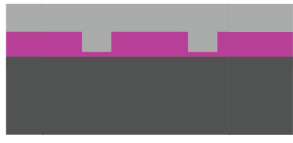

b)

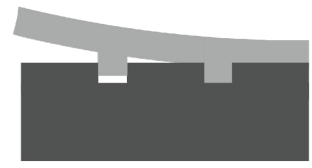

d)

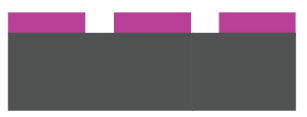

Figure 2. Process flow diagram of nanoimprint lithography process. (a) PDMS mixture is poured on the master stamp and takes its shape. (b) PDMS mold is cured and peeled off from the master. (c) PDMS mold is pressed on the wafer and patterns the resist. (d) Residual resist is removed from the undesired locations by dry etching.

E-beam lithography is a high resolution lithography technique which can resolve sub-100 $\mathrm{nm}$ features. Plasmonic nanoantennas with desired sizes were fabricated but the process was slow and costly. The contamination of the junction due to the residual e-beam resist (PMMA) was also suspected to degrade the device performance. The SEM image of the nanoantennas fabricated with e-beam lithography technique is shown in Fig. 3(b).
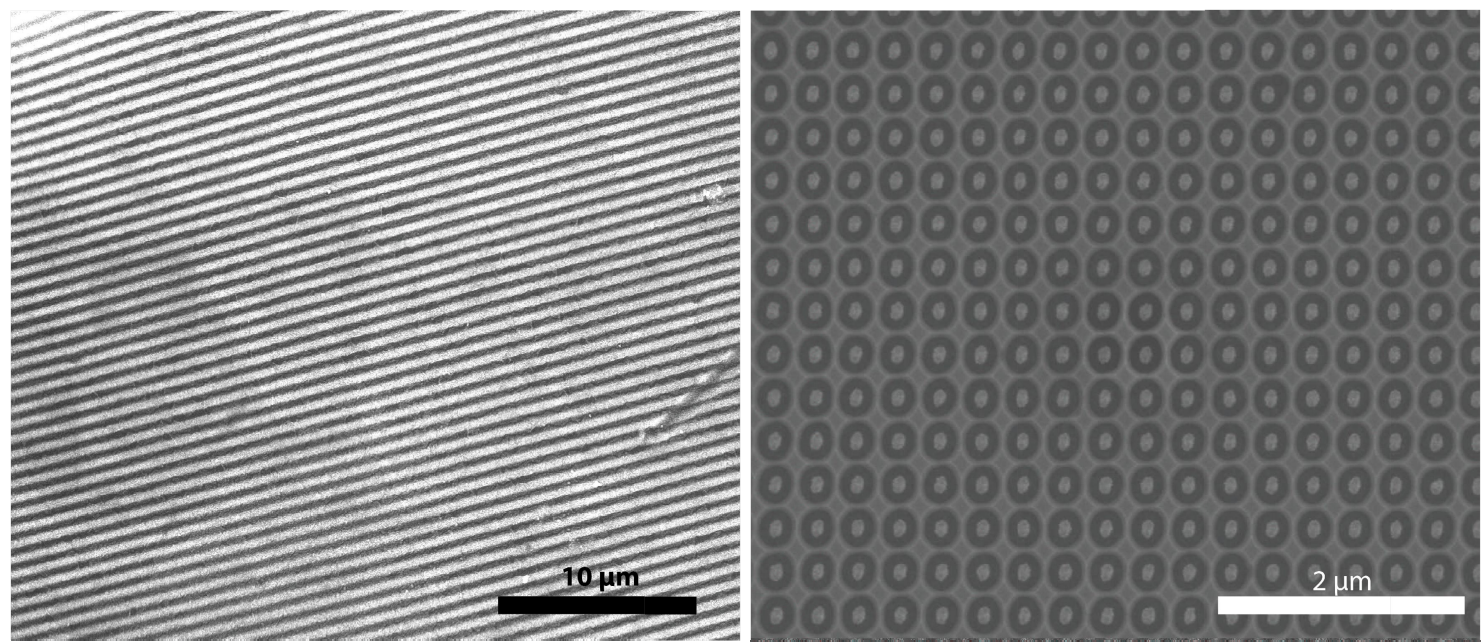

Figure 3. SEM images of the nanopatterned samples. (a) Grating structure obtained with nanoimprint lithography method. (b) Nanoantennas obtained with electron beam lithography, etching and lift-off.

Our final method for plasmonic nanoantenna fabrication involves rapid thermal annealing (RTA) of a thin metal film deposited on Si samples. Au and Ag were used in the experiments as the metal layer. Au is sputtered by using Gatan, Inc. Precision Etching Coating System (PECS) and Ag is thermally evaporated with VAKSIS MIDAS Thermal Evaporator. SRO-704 RTA system from ATV Technologie GmbH was used for rapid thermal annealing of the samples. The 
annealing was done by increasing the temperature from the room temperature to the desired set point and keeping the temperature constant at the set point for one minute. The system was then cooled down under $\mathrm{N}_{2}$ flow with no additional cooling. The experiments were made at three different annealing temperatures: $300^{\circ} \mathrm{C}, 450^{\circ} \mathrm{C}$ and $600^{\circ} \mathrm{C}$. When the metal layer is too thick or the annealing temperature is not sufficiently high the metal layer forms into connected nanoislands. A metal layer thickness of about $10 \mathrm{~nm}$ resulted in isolated nanoislands after annealing at high temperatures. Average size of the nanoislands depends on the RTA temperature, resulting in smaller nanoislands as the annealing temperature increases. This technique is significantly low-cost and easy to process compared to the high resolution lithography techniques investigated in this study. The contamination of the junction is also avoided since no photoresist or additional layer is used to obtain the nanoislands. The SEM images of the nanoislands obtained by annealing at different temperatures are shown in Fig. 4.
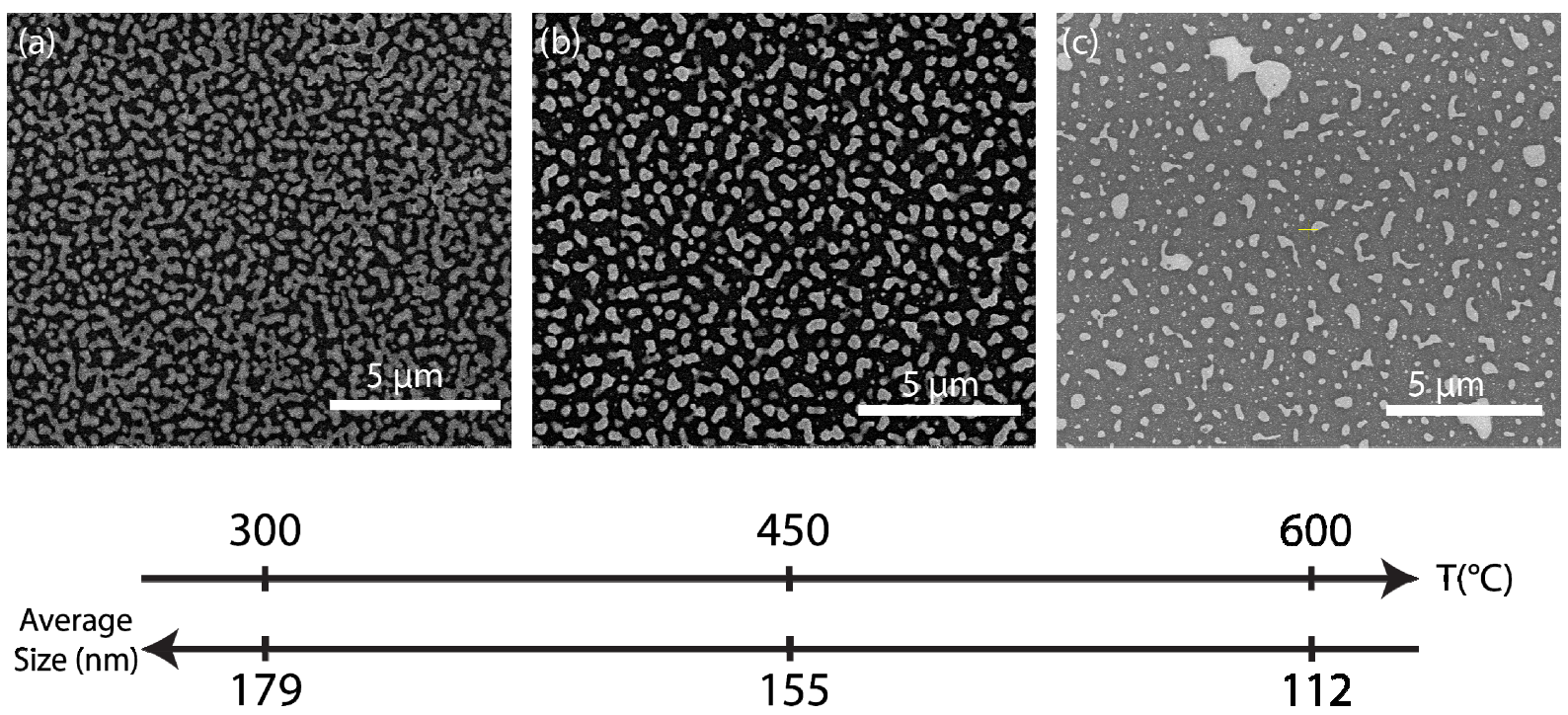

Figure 4. SEM image of the randomly sized and randomly distributed Au nanoislands obtained with rapid thermal annealing of a thin Au layer. Average particle size decreases with increasing temperature. If the annealing temperature is not sufficiently high the nanoislands are not isolated and form a semi-connected structure.

Annealing the $\mathrm{Ag}$ film at $300^{\circ} \mathrm{C}$ and $600^{\circ} \mathrm{C}$ resulted in connected and isolated $\mathrm{Ag}$ nanoislands, respectively. This result also indicates that average size of $\mathrm{Ag}$ nanoislands can be controlled with the annealing temperature. The plasmonic resonances of $\mathrm{Ag}$ nanoislands were at shorter wavelengths than the absorption band edge of Si due to higher plasmon frequency of $\mathrm{Ag}$ compared to $\mathrm{Au}$, which was later confirmed by finite-difference time-domain (FDTD) simulations. Although these nanoislands can possibly be used to enhance the absorption of $\mathrm{Si}$ at these wavelengths, it is difficult to observe hot electron based sub-bandgap photodetection with these Ag nanoislands.

FDTD simulations of Au nanoantennas on Si predicted strong plasmonic resonance in the NIR region of the spectrum and $\mathrm{Au}$ became the material of choice for the plasmonic nanoantennas in this study. Au nanoislands were fabricated by rapid thermal annealing at $300^{\circ} \mathrm{C}, 450^{\circ} \mathrm{C}$ and $600^{\circ} \mathrm{C}$. The particle size histograms were extracted from the SEM images by using the ImageJ software and the average particle sizes were calculated for each annealing temperature. By annealing at lower temperatures $\left(300^{\circ} \mathrm{C}\right)$ the average size of the nanoparticles increases and the plasmonic resonances are expected to shift to the longer wavelengths.

An Aluminum-doped Zinc Oxide (AZO) film is deposited on the Si wafer over the Au nanoislands to act as a transparent conducting oxide (TCO) layer. AZO layer is deposited in an atomic layer deposition (ALD) reactor (Cambridge Nanotech Inc., Savannah S100 ALD) at $250^{\circ} \mathrm{C}$ by alternating cycles of diethylzinc (DEZn) + milli-Q water $\left(\mathrm{H}_{2} \mathrm{O}\right)$ and trimethylaluminum (TMAl) + milli-Q water $\left(\mathrm{H}_{2} \mathrm{O}\right)$. DEZn cycle and TMAl cycle was repeated at a ratio of 24:1. $\mathrm{ZnO}$ deposited by ALD at $250^{\circ} \mathrm{C}$ is a transparent semiconductor and its conductive can be increased by doping it with Al. The AZO layer electrically connects the Au nanoislands at one contact of the device and also forms a heterojunction with $\mathrm{Si}^{5}$. 
AZO layer was then patterned with photolithography and nitric acid $\left(\mathrm{HNO}_{3}\right)$ etching. The devices were rinsed with DIwater after the etching step and were characterized without additional metallic contacts.

We fabricated three devices with Au nanoislands annealed at different temperatures $\left(300^{\circ} \mathrm{C}, 450^{\circ} \mathrm{C}\right.$ and $\left.600^{\circ} \mathrm{C}\right)$. Two additional devices were fabricated as references. The first reference only had the AZO layer forming a heterojunction with $\mathrm{Si}$, without $\mathrm{Au}$ nanoislands in between (AZO reference). The second reference only had a 10-nm-thick Au film on $\mathrm{Si}$, patterned in $300 \mu \mathrm{m} \times 600 \mu \mathrm{m}$ rectangles by using photolithography and lift-off method (Au reference). Since both the AZO layer and Si wafer are expected to be transparent in NIR wavelengths, the AZO reference is not expected to exhibit any photoresponse beyond the absorption band edge of Si. On the other hand, Au reference is expected to show photoresponse due to the presence of Au absorber. However, the efficiency of Au reference is expected to be low since the normally incident light will not excite surface plasmons on the flat Au layer. The photoresponses of the devices with $\mathrm{Au}$ nanoislands are expected to be much greater than the $\mathrm{Au}$ reference due to the plasmonic enhancement of hot electron generation process.

\section{RESULTS \& DISCUSSION}

For optoelectronic characterization of our photodetectors, we used Fianium Ltd, supercontinuum laser source (WLSC400-2), which provides high power and broad-band light from $390 \mathrm{~nm}$ to $2400 \mathrm{~nm}$ wavelength. The laser light is fed to a two-channel Fianium Ltd, acousto-optic tunable filter (AOTF) to monochromate the laser light at the desired wavelength. The monochromated laser light goes through an optical path with a mechanical chopper and a half-wave plate. Finally, a 20x-objective lens focuses the laser light normally on the photodetector. The beam waist of the focused light is approximately $50 \mu \mathrm{m}$. The photodetector is probed with micromanipulators and electrically biased with KEITHLEY 2401 Sourcemeter. The sourcemeter applies the bias voltage and measures the DC current. Photocurrent is measured with Stanford Research Systems SRS830 lock-in amplifier with the optical chopper frequency as the reference signal. A schematic drawing of the optoelectronic characterization setup is shown in Fig. 5.

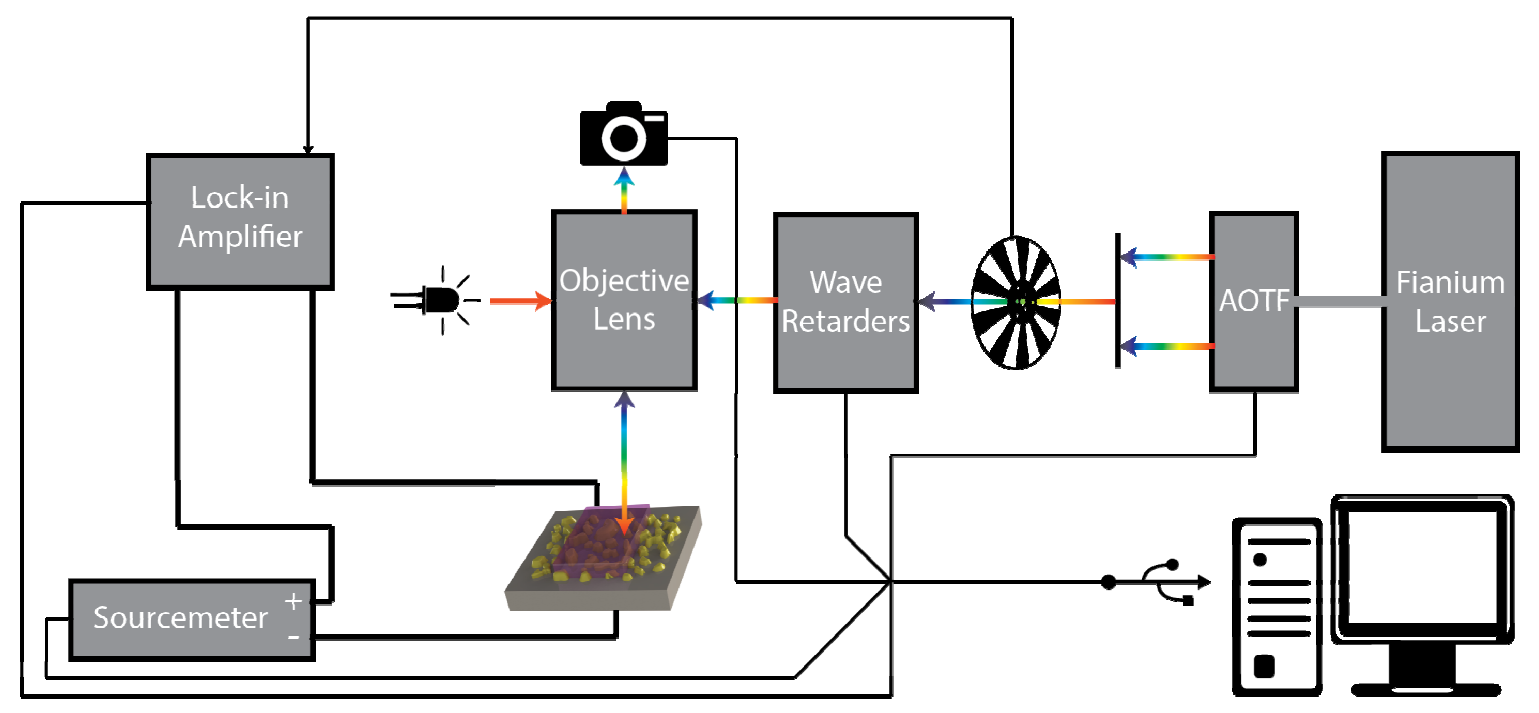

Figure 5. Schematic drawing of the optoelectronic characterization setup. Supercontinuum laser light and AOTF is used as the light source and modulated with a mechanical chopper. The light is focused on the devices with a custom optical setup. The photogenerated current at the frequency of the mechanical chopper is measured with a lock-in amplifier.

The quantum efficiency spectrum of the devices are plotted in Fig. 6(a). AZO reference does not respond to the NIR light above the absorption band edge of $\mathrm{Si}$. Au reference exhibits photoresponse at the NIR wavelengths but the photoresponsivity is much smaller than of the other devices. The device annealed at $450^{\circ} \mathrm{C}$ exhibits the best overall performance at the wavelength range of interest. The device annealed at $300^{\circ} \mathrm{C}$ has larger and more elongated nanoislands compared to the devices annealed at higher temperatures and the responsivity of this device is the highest at 
longer wavelengths $(\lambda>1500 \mathrm{~nm})$. Also the sample annealed at $600^{\circ} \mathrm{C}$ has smaller particles compared to the other samples and is expected to resonate at shorter wavelengths and exhibit lower response in NIR region.

Experimental quantum efficiency spectra were also compared with theoretically calculated quantum efficiency spectra in order to confirm the photocurrent generation via the plasmon enhanced internal photoemission process. SEM images of the $\mathrm{Au}$ nanoislands were imported to a Finite-Difference Time-Domain solver (FDTD Solutions, Lumerical Inc), assuming a uniform film thickness of $10 \mathrm{~nm}$. The absorption spectra of the Au nanoislands with different sizes and distributions are calculated. These absorption spectra correspond to hot electron generation rates in the Au nanoislands of each device, and need to be multiplied by the internal photoemission efficiency of hot electrons in order to calculate the theoretical quantum efficiency spectra. The efficiency of the internal photoemission process, which is expressed by the Fowler function, can be written as:

$$
\eta=C_{F} \frac{\left(h v-q \varphi_{B}\right)^{2}}{h v}
$$

where $\mathrm{C}_{\mathrm{F}}$ is the Fowler emission coefficient, hv is the energy of the incident photons, $\mathrm{q}$ is the electron charge and $\varphi_{\mathrm{B}}$ is the Schottky barrier height. The simulated result is then fitted to the experimental results with $\mathrm{C}_{\mathrm{F}}$ and $\varphi_{\mathrm{B}}$ used as the fit parameters. $\varphi_{\mathrm{B}}$ is approximately $0.7 \mathrm{eV}$ for each case, as expected from such junctions ${ }^{6}$. Simulated quantum efficiency spectra are plotted in Fig. 6(b). Simulated results are in good agreement with the experimental results, indicating that the sub-bandgap photodetection is achieved by the decay of surface plasmons to hot carriers and the collection of these hot carriers at the Schottky junction. The cross-over point of the quantum efficiency spectra of the devices annealed at $300^{\circ} \mathrm{C}$ and $450^{\circ} \mathrm{C}$ is also correctly predicted. This cross-over point occurs since the sample annealed at $300^{\circ} \mathrm{C}$ absorbs the longer wavelengths more effectively compared to the sample annealed at $450^{\circ} \mathrm{C}$. Hence, at this cross-over point, the efficiency of the sample annealed at $300^{\circ} \mathrm{C}$ exceeds that of the sample annealed at $450^{\circ} \mathrm{C}$. The sample annealed at $600^{\circ} \mathrm{C}$ has weaker response in NIR since its smaller particles resonate at shorter wavelengths.

a)

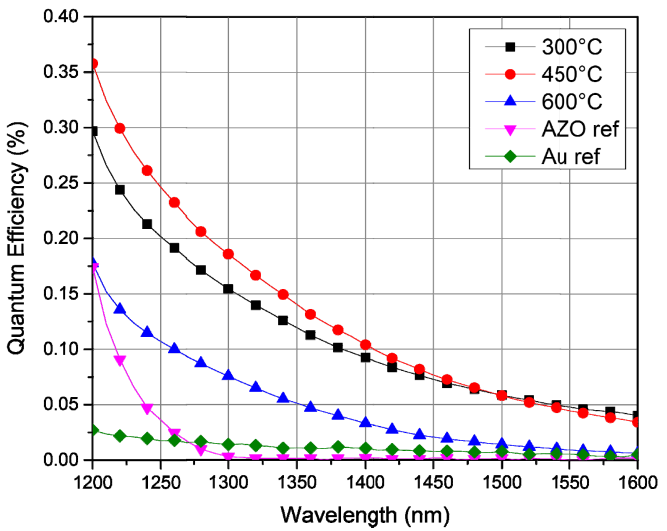

b)

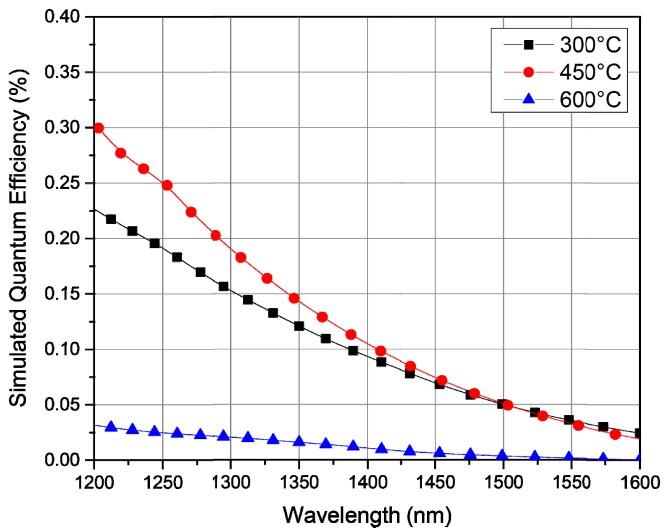

Figure 6. (a) Measured quantum efficiency spectra of the reference devices and the devices with nanoislands formed by rapid thermal annealing at different temperatures. (b) Simulated quantum efficiency spectra of the nanoislands formed by rapid thermal annealing at different temperatures. Absorption spectra of the nanoislands in our device configuration are obtained with FDTD simulations. Quantum efficiency spectra are calculated by multiplying the simulated absorption spectra with the Fowler function and fitting to the experimental results with the correct coefficients of the Fowler function.

\section{CONCLUSION}

The enhancement of hot electron generation rate by the excitation of surface plasmons on metals is investigated as an efficient and low-cost method for the detection of sub-bandgap photons on Si. Among various techniques to fabricate plasmonic nanostructures, rapid thermal annealing a thin metal film stand out as a fast and ultra-low-cost method to obtain random nanostructures with strong, broad-band resonances at the NIR wavelengths. With the proposed device structure, up to $0.18 \%$ and $0.05 \%$ quantum efficiencies were observed at $1300 \mathrm{~nm}$ and $1550 \mathrm{~nm}$ wavelengths, 
respectively. FDTD simulations and theoretical calculations were used to fit to the experimental results and successfully predict the quantum efficiency spectrum.

\section{ACKNOWLEDGMENTS}

This work was supported by the Scientific and Technological Research Council of Turkey (TUBITAK), grant numbers 109E044, 112M004, 112E052, and 113M815. A. K. O. acknowledges support from European Union FP7 Marie Curie International Reintegration Grant (PIOS, Grant \# PIRG04-GA-2008-239444). A. K. O. acknowledges support from the Turkish Academy of Sciences Distinguished Young Scientist Award (TUBA GEBIP). F. B. A. acknowledges TUBITAK-BIDEB national PhD Fellowship.

\section{REFERENCES}

[1] Peters, D. W., "An infrared detector utilizing internal photoemission," Proceedings of the IEEE 55, 704-705 (1967).

[2] Knight, M. W., Sobhani, H., Nordlander, P. and Halas, N. J., "Photodetection with active optical antennas," Science 332, $702-704$ (2011).

[3] Sobhani, A., Knight, M. W., Wang, Y., Zheng, B., King, N. S., Brown, L. V., Fang, Z., Nordlander, P. and Halas, N. J., "Narrowband photodetection in the near-infrared with a plasmon-induced hot electron device," Nature communications 4, 1643 (2013).

[4] Nazirzadeh, M. A., Atar, F. B., Turgut, B. B., Okyay, A. K., "Random sized plasmonic nanoantennas on Silicon for low-cost broad-band near-infrared photodetection," Scientific Reports 4, 7103 (2014).

[5] Ismail, R. A., Al-Naimi, A. and Al-Ani, A. A., "Studies on fabrication and characterization of a high-performance Al-doped $\mathrm{ZnO} / \mathrm{n}-\mathrm{Si}\left(\begin{array}{lll}1 & 1 & 1\end{array}\right)$ heterojunction photodetector," Semiconductor Science and Technology 23, 075030 (2008).

[6] Sze, S. M., and Ng, K. K., [Physics of semiconductor devices], John Wiley \& Sons (2006). 